QUARTERLY OF APPLIED MATHEMATICS

VOLUME LXX, NUMBER 1

MARCH 2012, PAGES 69-76

S 0033-569X(2011)01232-3

Article electronically published on August 26, 2011

\title{
STABILITY OF ZND DETONATIONS FOR MAJDA'S MODEL
}

\author{
BY \\ SOYEUN JUNG (Indiana University, Bloomington, Indiana 47405) \\ AND \\ JINGHUA YAO (Indiana University, Bloomington, Indiana 47405)
}

\begin{abstract}
We evaluate by direct calculation the Lopatinski determinant for ZND detonations in Majda's model for reacting flow and show that on the nonstable (nonnegative real part) complex half-plane it has a single zero at the origin of multiplicity one, implying stability. Together with results of Zumbrun on the inviscid limit, this recovers the result of Roquejoffre-Vila that viscous detonations of Majda's model also are stable for sufficiently small viscosity, for any fixed detonation strength, heat release, and rate of reaction.
\end{abstract}

1. Introduction. In this note, we verify by explicit computation the spectral stability in the sense of Erpenbeck [Er] of strong ZND detonation wave solutions of Majda's model for reactive gas dynamics $[\mathrm{M}]$ with a step-type ignition function.

Consider the inviscid Majda's model

$$
\begin{aligned}
(u+q z)_{t}+\left(\frac{u^{2}}{2}\right)_{x} & =0, \\
z_{t}+k \varphi(u) z & =0,
\end{aligned}
$$

an analog of the Zeldovich-Von Neumann-Doering (ZND) equations for reactive gas dynamics, where $u, z, \varphi \in \mathbb{R}, q \geq 0$ and $k>0$. Here, $u$ is a lumped variable modeling the gas-dynamical quantities of density, momentum, and energy, $z$ is the mass fraction of the reactant, $q \geq 0$ a coefficient of heat release of the reaction, $k>0$ reaction rate, and $\varphi(u)$ is a simple step-type "ignition function" that is assumed to be zero below a certain value $u_{i}>0$ and one above.

A strong detonation wave of (1.1) is a traveling-wave solution

$$
(u, z)(x, t)=(\bar{u}, \bar{z})(x-s t), \quad \lim _{\xi \rightarrow \pm \infty}(\bar{u}, \bar{z})(\xi)=\left(u_{ \pm}, z_{ \pm}\right)
$$

Received May 21, 2010.

2000 Mathematics Subject Classification. Primary 76L05; Secondary 76E99, 76N99, 80A32.

The research of S.J. and J.Y. was partially supported under NSF grants number DMS-0070765 and DMS-0300487. Thanks to Kevin Zumbrun for suggesting the problem and for helpful discussions.

E-mail address: soyjung@indiana.edu

E-mail address: yaoj@indiana.edu

(C)2011 Brown University

Reverts to public domain 28 years from publication 
in the weak, or distributional, sense, smooth except at a single shock discontinuity at (without loss of generality) $x=0$, known as a "Neumann shock", where $u$ jumps from $u_{*}:=\bar{u}\left(0^{-}\right)$to $\bar{u}\left(0^{+}\right)$as $x$ crosses zero from left to right, and satisfying

$$
z_{-}=0, z_{+}=1, \quad u_{-}>u_{i}>u_{+}
$$

and

$$
u_{-}>s>u_{+} .
$$

Computing the Rankine-Hugoniot conditions at the shock at $x=0$, we find that

$$
\begin{aligned}
s\left(\bar{u}\left(0^{+}\right)-\bar{u}\left(0^{-}\right)\right) & =\frac{\bar{u}\left(0^{+}\right)^{2}}{2}-\frac{\bar{u}\left(0^{-}\right)^{2}}{2}, \\
\bar{z}\left(0^{-}\right) & =\bar{z}\left(0^{+}\right) .
\end{aligned}
$$

By the various invariances of equations (1.1), we may take without loss of generality

$$
u_{*}=2, \quad u_{+}=0, \quad s=1,
$$

letting $u_{-}, q>0, k>0$ vary. From (1.4) and the assumption that $(\bar{u}, \bar{z})$ converges as $x \rightarrow \pm \infty$, we find further by consideration of the traveling-wave ODE (see Section 2) that

$$
(\bar{u}, \bar{z})(x) \equiv\left(u_{+}, z_{+}\right)=(0,1) \text { for } x>0
$$

and also $u_{-}=1+\sqrt{1-2 q}$, so that

$$
1 \leq u_{-} \leq 2, \quad 0 \leq q \leq \frac{1}{2},
$$

with reaction rate varying in the infinite range $0<k<+\infty$.

That is, we have the standard picture of a strong detonation wave as a shock advancing to the right into a quiescent (i.e., nonreacting) constant state with reactant mass fraction $z=1$, raising $u$ above the ignition level $u_{i}$, followed by a smooth "reaction tail" in which combustion (reaction) occurs, in which $z$ decays exponentially to the value $z_{-}=0$ and $u$ to $1 \leq u_{-}<u_{*}$ as $x \rightarrow-\infty$. See [LyZ1, LyZ2, JLW, Z1, Z2, for further details.

As shown by Erpenbeck [Er, spectral stability of such waves, defined as nonexistence of normal modes $e^{\lambda t} w(x)$ with $\Re \lambda \geq 0$ and $\lambda \neq 0$, may be determined by examination of a certain Lopatinski determinant $D_{Z N D}(\lambda)$ whose zeros on $\Re \lambda \geq 0$ correspond to eigenvalues $\lambda$. Precisely, spectral stability corresponds to the condition

$$
D_{Z N D}(\cdot) \text { has a single zero on }\{\Re \lambda \geq 0\} \text { occurring at } \lambda=0 \text {. }
$$

The Lopatinski condition (D) has been much studied numerically in both one dimension and multi-dimensions, for the full equations of reactive gas dynamics; see, for example, $[\mathrm{KS}]$ and the references therein. However, so far as we know, it has up to now not been verified analytically for any case. Here, we show by direction calculation that (D) holds for detonations of the inviscid Majda model with step-type ignition function.

Since linear or spectral stability concerns only behavior near values of the profile $(\bar{u}, \bar{z})$, it is clear that this result extends also to the slightly more general class of (possibly smooth) ignition functions identified by Roquejoffre and Vila [RV] that $\phi(u) \equiv 0$ for 
$u<u_{i}$ and $\phi(u) \equiv 1$ for $u>u^{i}$, provided that $u_{-}>u^{i}>u_{i}>u_{+}$. That is, we require in our analysis only that

$$
\phi(u)=0 \text { near } u_{+} \text {and } \phi(u)=1 \text { near }\left[u_{-}, u_{*}\right] .
$$

A very interesting open problem is whether a corresponding stability result holds for more general ignition functions satisfying only

$$
\phi(u)=0 \text { for } u<u_{i} \text { and } \phi(u)>0 \text { for } u>u_{i} .
$$

As discussed in Section 6, these results have implications also for spectral and nonlinear stability of viscous detonation waves in the ZND limit as viscosity goes to zero.

2. Profile solutions. Observing that

$$
\partial_{t} \bar{u}(x-s t)=-s \bar{u}^{\prime}, \quad \partial_{x} \bar{u}(x-s t)=\bar{u}^{\prime}, \quad \partial_{t} \bar{z}(x-s t)=-s \bar{z}^{\prime}, \quad \partial_{x} \bar{z}(x-s t)=\bar{z}^{\prime},
$$

we obtain for a detonation solution (1.2) $-(1.6)$ the profile equations

$$
\begin{aligned}
-s(\bar{u}+q \bar{z})^{\prime}+\left(\frac{\bar{u}^{2}}{2}\right)^{\prime} & =0, \\
-s \bar{z}^{\prime}+k \varphi(\bar{u}) \bar{z} & =0,
\end{aligned}
$$

valid on the regions of smoothness $x>0$ and $x<0$. Linearizing (2.1) about the equilibrium solution $(u, z) \equiv\left(u_{+}, 1\right)$, we readily find from $s>u_{+}$that this is a repelling equilibrium, and so the only smooth solution of (2.1) converging to this equilibrium is the constant solution (1.7).

Integrating (2.1) from $-\infty$ to $+\infty$, we obtain

$$
s\left(u_{+}+q z_{+}\right)-\frac{u_{+}^{2}}{2}=s\left(u_{-}+q z_{-}\right)-\frac{u_{-}^{2}}{2} .
$$

Substituting $s=1,\left(u_{+}, z_{+}\right)=(0,1), z_{-}=0$, we find that

$$
-\bar{u}_{-}+q=-\frac{\bar{u}_{-}^{2}}{2} \text {. }
$$

Now, integrating (2.1) from $-\infty$ to $\xi$, we obtain

$$
-s(\bar{u}+q \bar{z})+s\left(\bar{u}_{-}+q \bar{z}_{-}\right)=-\frac{\bar{u}^{2}}{2}+\frac{\bar{u}_{-}^{2}}{2},
$$

from which, by (1.3), (2.2), and $s=1$, we obtain

$$
\bar{u}(\xi)=1 \pm \sqrt{1-2 q(1-\bar{z}(\xi))} .
$$

We choose the sign + because $u_{*}=2$, that is, 2 is the left-limit at $\xi=0$, and $\bar{z}(0)=1$, yielding in particular $u_{-}=1+\sqrt{1-2 q}$. Since $\varphi(\bar{u})=1$ for $\xi<0$ in (2.1), $\bar{z}^{\prime}=k \bar{z}$; hence for $\xi<0$,

$$
\bar{z}(\xi)=\bar{z}(0) e^{k \xi}=e^{k \xi}
$$

and

$$
\bar{u}(\xi)=1+\sqrt{1-2 q\left(1-e^{k \xi}\right)} .
$$


3. Eigenvalue equations. Let $(\tilde{u}, \tilde{z})$ be a solution of (1.1) different from $(\bar{u}, \bar{z})$. Subtracting $(\tilde{u}, \tilde{z})$ from $(\bar{u}, \bar{z})$, we obtain for the perturbation variable

$$
(u, z):=(\tilde{u}, \tilde{z})-(\bar{u}, \bar{z})
$$

the perturbation equations

$$
\begin{aligned}
(u+q z)_{t}+\left(\frac{\tilde{u}^{2}}{2}-\frac{\bar{u}^{2}}{2}\right)_{\xi} & =0, \\
z_{t}+k(\varphi(\tilde{u}) \tilde{z}-\varphi(\bar{u}) \bar{z}) & =0 .
\end{aligned}
$$

Taylor expanding and dropping $O\left(|(u, z)|^{2}\right)$ terms, we obtain the linearized equations

$$
\begin{aligned}
u_{t}-s u_{\xi}+(\bar{u} u)_{\xi} & =q k(d \varphi(\bar{u}) \bar{z} u+\varphi(\bar{u}) z), \\
z_{t}-s z_{\xi} & =-k(d \varphi(\bar{u}) \bar{z} u+\varphi(\bar{u}) z),
\end{aligned}
$$

and, finally, the linearized eigenvalue equations

$$
\begin{aligned}
\lambda u-s u^{\prime}+(\bar{u} u)^{\prime} & =q k(d \varphi(\bar{u}) \bar{z} u+\varphi(\bar{u}) z), \\
\lambda z-s z^{\prime} & =-k(d \varphi(\bar{u}) \bar{z} u+\varphi(\bar{u}) z),
\end{aligned}
$$

where $/$ denotes $\partial_{\xi}$.

Expanding and rearranging, we have

$$
\begin{aligned}
(\bar{u}-s) u^{\prime}+(\bar{u}-s)^{\prime} u & =(q k d \varphi(\bar{u}) \bar{z}-\lambda) u+q k \varphi(\bar{u}) z, \\
-s z^{\prime} & =-k d \varphi(\bar{u}) \bar{z} u+(-k \varphi(\bar{u})-\lambda) z,
\end{aligned}
$$

which give the matrix equation

$$
\begin{aligned}
\left\{\left(\begin{array}{cc}
\bar{u}-s & 0 \\
0 & -s
\end{array}\right)\left(\begin{array}{l}
u \\
z
\end{array}\right)\right\}^{\prime} & =\left(\begin{array}{cc}
q k d \varphi(\bar{u}) \bar{z}-\lambda & q k \varphi(\bar{u}) \\
-k d \varphi(\bar{u}) \bar{z} & -k \varphi(\bar{u})-\lambda
\end{array}\right)\left(\begin{array}{l}
u \\
z
\end{array}\right) \\
& =\left\{\left(\begin{array}{cc}
q k d \varphi(\bar{u}) \bar{z} & q k \varphi(\bar{u}) \\
-k d \varphi(\bar{u}) \bar{z} & -k \varphi(\bar{u})
\end{array}\right)-\lambda I\right\}\left(\begin{array}{l}
u \\
z
\end{array}\right) .
\end{aligned}
$$

Setting

$$
A=\left(\begin{array}{cc}
\bar{u}-s & 0 \\
0 & -s
\end{array}\right), W=\left(\begin{array}{c}
u \\
z
\end{array}\right), E=\left(\begin{array}{cc}
q k d \varphi(\bar{u}) \bar{z} & q k \varphi(\bar{u}) \\
-k d \varphi(\bar{u}) \bar{z} & -k \varphi(\bar{u})
\end{array}\right), Z=A W,
$$

we may write the eigenvalue equations as the first-order ODE system

$$
Z^{\prime}=(E-\lambda I) W=(E-\lambda I) A^{-1} Z=G Z,
$$

where $G=(E-\lambda I) A^{-1}$.

4. The Lopatinski determinant. Following [JLW, Z2, define on $\Re \lambda \geq 0$ the Lopatinski determinant

$$
D_{Z N D}(\lambda):=\operatorname{det}\left(Z^{-}(\lambda, 0), \lambda[\bar{W}]-\left[A \bar{W}^{\prime}\right]\right),
$$

where $[h]:=h\left(0^{+}\right)-h\left(0^{-}\right)$and $Z^{-}(\lambda, \xi)$ is a bounded exponentially decaying solution of (3.2), analytic in $\lambda$ and tangent as $\xi \rightarrow-\infty$ to the subspace of exponentially decaying solutions of the limiting, constant-coefficient equations $Z^{\prime}=G_{-} Z$. By standard asymptotic ODE theory (the "gap lemma" GZ), $Z^{-}$is uniquely determined up to a nonvanishing analytic factor not affecting stability. 
LEMma 4.1. The ZND Lopatinski determinant is given (up to a nonvanishing analytic factor) by

$$
\begin{gathered}
D_{Z N D}=(2 \lambda+(2-q-q k \Psi) k)\left(\frac{\lambda}{k+\lambda}\right), \\
\Psi:=\int_{-\infty}^{0} e^{-\int_{y}^{0} P(s) d s} \frac{e^{(k+\lambda) y}}{\sqrt{1-2 q\left(1-e^{k y}\right)}} d y, \quad P(\xi):=\frac{\lambda}{\sqrt{1-2 q\left(1-e^{k \xi}\right)}} .
\end{gathered}
$$

Proof. Combining (2.4), (2.5) and (3.1), we obtain

$$
\begin{aligned}
\lambda[\bar{W}]-\left[A\left(\bar{W}^{\prime}\right)\right]=\lambda\left[\left(\begin{array}{c}
\bar{u} \\
\bar{z}
\end{array}\right)\right]+\left(A \bar{W}^{\prime}\right)\left(0^{-}\right) & =\lambda\left(\begin{array}{c}
-2 \\
0
\end{array}\right)+\left(\begin{array}{cc}
1 & 0 \\
0 & -1
\end{array}\right)\left(\begin{array}{c}
q k \\
k
\end{array}\right) \\
& =\left(\begin{array}{c}
-2 \lambda+q k \\
-k
\end{array}\right),
\end{aligned}
$$

where we are using $\bar{W}^{\prime}=\left(\frac{q k e^{k \xi}}{\sqrt{1-2 q\left(1-e^{k \xi}\right)}}, k e^{k \xi}\right)^{T}$. Computing

$$
G=(E-\lambda I) A^{-1}=\left(\begin{array}{cc}
-\lambda & q k \\
0 & -k-\lambda
\end{array}\right)\left(\begin{array}{cc}
\bar{u}-1 & 0 \\
0 & -1
\end{array}\right)^{-1}=\left(\begin{array}{cc}
\frac{-\lambda}{\bar{u}-1} & -q k \\
0 & k+\lambda
\end{array}\right)
$$

and setting $Z=\left(\begin{array}{c}Z_{1} \\ Z_{2}\end{array}\right)$, we have

$$
\left(\begin{array}{c}
Z_{1}(\lambda, \xi) \\
Z_{2}(\lambda, \xi)
\end{array}\right)^{\prime}=\left(\begin{array}{cc}
\frac{-\lambda}{\bar{u}-1} & -q k \\
0 & k+\lambda
\end{array}\right)\left(\begin{array}{c}
Z_{1}(\lambda, \xi) \\
Z_{2}(\lambda, \xi)
\end{array}\right)
$$

or

$$
\begin{aligned}
Z_{1}^{\prime} & =-\frac{\lambda}{\bar{u}-1} Z_{1}-q k Z_{2} \\
Z_{2}^{\prime} & =(k+\lambda) Z_{2} .
\end{aligned}
$$

Solving, we obtain

$$
\begin{aligned}
Z_{1}^{\prime} & =-\frac{\lambda}{\sqrt{1-2 q\left(1-e^{k \xi}\right)}} Z_{1}-q k e^{(k+\lambda) \xi} \\
Z_{2} & =e^{(k+\lambda) \xi}
\end{aligned}
$$

Now setting $P(\xi):=\frac{\lambda}{\sqrt{1-2 q\left(1-e^{k \xi}\right)}}$ and $Q(\xi):=-q k e^{(k+\lambda) \xi}$, we have

$$
\int P(\xi) d \xi=-\frac{2 \lambda}{k \sqrt{1-2 q}} \ln \left(\frac{\sqrt{1-2 q\left(1-e^{k \xi}\right)}+\sqrt{1-2 q}}{\sqrt{2 q e^{k \xi}}}\right),
$$


which gives $\left|e^{-\int_{-\infty}^{0} P(\xi) d \xi}\right| \leq 1$ for $\Re \lambda \geq 0$, and hence, integrating by parts,

$$
\begin{aligned}
Z_{1}(\lambda, 0) & =e^{-\int_{-\infty}^{0} P(s) d s}\left(\int_{-\infty}^{0} e^{\int_{-\infty}^{y} P(s) d s} Q(y) d y\right) \\
& =\int_{-\infty}^{0} e^{-\int_{y}^{0} P(s) d s} Q(y) d y \\
& =-\left.e^{-\int_{y}^{0} P(s) d s} \frac{q k}{k+\lambda} e^{(k+\lambda) y}\right|_{-\infty} ^{0}+\int_{-\infty}^{0} e^{-\int_{y}^{0} P(s) d s} P(y) \frac{q k}{k+\lambda} e^{(k+\lambda) y} d y \\
& =-\frac{q k}{k+\lambda}(1-\lambda \Psi)
\end{aligned}
$$

where $\Psi$ is as in (4.3). By (4.1), (4.4), and (4.5),

$$
\begin{aligned}
D_{Z N D}(\lambda) & =\operatorname{det}\left(Z^{-}(\lambda, 0), \lambda[\bar{W}]+A\left(\bar{W}^{\prime}\right)\left(0^{-}\right)\right) \\
& =\operatorname{det}\left(\begin{array}{cc}
Z_{1}(\lambda, 0) & -2 \lambda+q k \\
Z_{2}(\lambda, 0) & -k
\end{array}\right) \\
& =-k Z_{1}+2 \lambda-q k \\
& =\frac{q k^{2}(1-\lambda \Psi)+(2 \lambda-q k)(k+\lambda)}{k+\lambda} \\
& =\frac{-q k^{2} \lambda \Psi+2 k \lambda+2 \lambda^{2}-q k \lambda}{k+\lambda} \\
& =(2 \lambda+(2-q-q k \Psi) k)\left(\frac{\lambda}{k+\lambda}\right) .
\end{aligned}
$$

\section{Verification of spectral stability.}

Theorem 5.1. For a step-type ignition function, or, more generally, any ignition function satisfying (1.9), $D_{Z N D}$ has a single zero of multiplicity one on $\{\Re \lambda \geq 0\}$, located at $\lambda=0$; that is, the Lopatiski condition (D) is satisfied for all ZND detonations of Majda's model, independent of the choice of $q \geq 0, k>0$, or $u_{ \pm}$.

Proof. By (4.3), we have for $\Re \lambda \geq 0$,

$$
\begin{aligned}
|\Psi| & \leq \int_{-\infty}^{0} e^{-\int_{y}^{0} \Re P(s) d s} \frac{e^{(k+\Re \lambda) y}}{\sqrt{1-2 q\left(1-e^{k y}\right)}} d y \\
& \leq \int_{-\infty}^{0} \frac{e^{k y}}{\sqrt{1-2 q\left(1-e^{k y}\right)}} d y \\
& =\frac{1}{q k} \int_{-\infty}^{0} \frac{d}{d y}\left(\sqrt{1-2 q\left(1-e^{k y}\right)}\right) d y \\
& =\frac{1-\sqrt{1-2 q}}{q k}
\end{aligned}
$$

giving

$$
\Re(2-q-q k \Psi) \geq 2-q-q k|\Psi| \geq 1-q+\sqrt{1-2 q}>0
$$


by the fact (see (1.8)) that $0 \leq q \leq \frac{1}{2}$. In particular,

$$
2-q-q k \Psi \neq 0 \text { for } \Re \lambda \geq 0 .
$$

Combining (4.6) and (5.1), we obtain the result.

6. Viscous stability and a result of Roquejoffre-Vila. Our results have implications also for stability of "viscous" detonation waves, i.e., smooth analogs of traveling waves (1.2) satisfying the "viscous" or parabolic regularization of (1.1):

$$
\begin{aligned}
(u+q z)_{t}+\left(\frac{u^{2}}{2}\right)_{x} & =\epsilon q z_{x x}+\epsilon u_{x x} \\
z_{t}+k \varphi(u) z & =\epsilon z_{x x}
\end{aligned}
$$

with $\epsilon>0$. It is shown in Z2 that stability of viscous detonation waves in the ZND limit as $\epsilon \rightarrow 0$ with other parameters held fixed is equivalent to Lopatinski stability of the limiting ZND detonation (1.2) together with Evans (equivalently, linearized) stability of the viscous Burgers shock corresponding to the Neumann shock contained in the ZND detonation profile, as is well known to hold by properties of scalar traveling-waves (see, e.g., $\mathrm{Sa}]$.

Thus, our results together with those of [Z2] yield the result for (6.1) of spectral stability of viscous detonation waves in the ZND limit, similar to an earlier result of Roquejoffre-Vila [RV] for the corresponding equations with regularization $\epsilon u_{x x}$ in the $u$ equation alone and applying to the same class of ignition functions (1.9). A very interesting open problem would be to extend our results to the more general class of ignition functions (1.10), which would give new information for the viscous stability problem as well.

We remark that results of [LRTZ] (for Majda's model) and [TZ] (for the physical reactive Navier-Stokes equations) show that spectral stability of viscous detonation waves in the (Evans function) sense of [Z2] implies linearized and nonlinear orbital stability; hence stability of strong viscous detonation waves reduces for viscosity sufficiently small to a study of spectral ZND stability as carried out here.

\section{REFERENCES}

[Er] J. J. Erpenbeck, Stability of idealized one-reaction detonations, Phys. Fluids 7 (1964).

[GZ] R. Gardner and K. Zumbrun, The Gap Lemma and geometric criteria for instability of viscous shock profiles. Comm. Pure Appl. Math. 51 (1998), no. 7, 797-855. MR.1617251 (99c:35152)

[JLW] H.K. Jenssen, G. Lyng, and M. Williams. Equivalence of low-frequency stability conditions for multidimensional detonations in three models of combustion, Indiana Univ. Math. J. 54 (2005), 1-64. MR2126075 (2006a:35249)

[KS] A.R. Kasimov and D.S. Stewart, Spinning instability of gaseous detonations. J. Fluid Mech. 466 (2002), 179-203. MR1925152 (2003g:76093)

[LyZ1] G. Lyng and K. Zumbrun, One-dimensional stability of viscous strong detonation waves, Arch. Ration. Mech. Anal. 173 (2004), no. 2, 213-277. MR2081031(2005f:76061)

[LyZ2] G. Lyng and K. Zumbrun, A stability index for detonation waves in Majda's model for reacting flow, Physica D, 194 (2004), 1-29. MR2075662 (2005d:35134)

[LRTZ] G. Lyng, M. Raoofi, B. Texier, and K. Zumbrun, Pointwise Green Function Bounds and stability of combustion waves, J. Differential Equations 233 (2007), 654-698. MR2292522 (2007m:35147)

[M] A. Majda, A qualitative model for dynamic combustion, SIAM J. Appl. Math., 41 (1981), 70-91. $\operatorname{MR} 622874(82 \mathrm{j}: 35096)$ 
[RV] J.-M. Roquejoffre and J.-P. Vila, Stability of ZND detonation waves in the Majda combustion model, Asymptot. Anal. 18 (1998), no. 3-4, 329-348. MR.1668958 (99m:80016)

[Sa] D. Sattinger, On the stability of waves of nonlinear parabolic systems. Adv. Math. 22 (1976), 312-355. MR0435602 (55:8561)

[TZ] B. Texier and K. Zumbrun, Transition to longitudinal instability of detonation waves is generically associated with Hopf bifurcation to time-periodic galloping solutions, preprint (2008).

[Z1] K. Zumbrun, Multidimensional stability of planar viscous shock waves, Advances in the theory of shock waves, 307-516, Progr. Nonlinear Differential Equations Appl., 47, Birkhäuser Boston, Boston, MA, 2001. MR1842778 (2002k:35200)

[Z2] K. Zumbrun, Stability of viscous detonation waves in the ZND limit, preprint (2009). 